\title{
The Application Of Microcapsules Of PCM In Flame Resistant Non-Woven Materials
}

\author{
Isabel Cardoso \\ Micropolis SA, Braga, Portugal, and \\ Jaime Rocha Gomes \\ Department of Textile Engineering, University of Minho, Portugal
}

\begin{abstract}
Purpose-The use of organic phase change materials microcapsules (mPCM) has been gaining ground in technical textiles as a temperature regulating medium and hence a means of keeping the body at a comfortable temperature when wearing impermeable protective clothes. However, for such applications as fire fighter's protective clothes, the standards require that all the material composing the material be fire resistant. The purpose of this work is to produce a lining containing fire resistant microcapsules of PCM without using flammable binders.

Methodology - In this work other ways of fixing MPCM to the fibres with a lot less binder present were tested. Washfastness was evaluated in SEM photographs and by weight. The thermal effect was evaluated in a prototype plate calorimeter.

Findings - This method was first tested for fixing $\mathrm{MPCM}$ but the non-woven still didn't pass the test according to the standard EN532. Microcapsules were alternatively fixed with MF resin, non-flammable, and by applying flame retardant recipes it was possible for the samples to pass the test.

Research limitations - Since the amount of flame retardant necessary for the MPCM to stand the test, and the resin to thermo fix it was very high, the material became unacceptably stiff.

Originality -Based on a new approach where reactive microcapsules without any binder were used, it was possible to use a lot less flame retardant and resin, and the material was resistant to the standard EN532. In this standard the material has to resist washing and still be flame retardant.
\end{abstract}

Key words- Microcapsules, PCM, flame retardant, thermal comfort, protection clothes

\section{INTRODUCTION}

Protection clothes for firefighters are made in a way that they protect them from external heat. The materials used are usually thick and heavy so as to provide the necessary insulation to heat. Whereas they perform as expected during the firefighting, the energy the firefighter spends on the exercise of firefighting and accessory tasks, aggravated by the fact that the firefighting clothes are heavy and non-breathable, makes him experience discomfort and extra stress. The use of organic microcapsules of phase change materials (mPCM) has been gaining ground in technical textiles as a temperature regulating medium and hence as a means of keeping the body at a comfortable temperature when wearing impermeable protective clothes. They work by absorbing the extra energy the body releases, and then giving it back when the body cools down. This exchange of energy happens around the phase change temperature (melting point) of the PCM which is this case is $28^{\circ} \mathrm{C}$, which is also considered the comfort temperature for the body. However, for such applications as fire fighter's protective clothes, the standards require that all the material composing the material be fire resistant. Since PCM are flammable paraffins, they should be microencapsulated in heat resistant material so that they don't become exposed to the flames due to deterioration of the microcapsule shell. The most common material used as shell material is melamine-formaldehyde, since it is heat resistant. It is also in itself a fairly good flame resistant material[1,2],

In this work the mPCM had to be applied to a non-woven material made of aramid fibre, which is a flame resistant, in such a way as not to bind it with flammable binders. Most applications of microcapsules are with thermoplastic binders such as acrylic or polyurethane. In $\mathrm{s}$ first attempt to avoid this problem, instead of immersing the microcapsules in a binder, the microcapsules 
were coated with a second wall made of binder material[3], acrylates, and this second wall was treated with flame retardant products. To fix the microcapsules and the fire retardant a crosslinking resin, also based on melamine-formaldehyde was used, which altered the non-woven material making it too stiff. Other solutions were tested such as the direct binding of the microcapsules with melamine-formaldehyde resins without the use of acrylate thermoplastic binders and the application of alternative fire retardant products. The different fire retardant products tested were of different types, and chosen so as to bind onto the microcapsules or/and bind onto the material. In the first case a halogenated copolymer was used on the coating of the MF mPCM for one trial and a metal oxide was bound by ion exchange to a polymer coated onto the MF mPCM on another trial. For the non coated MPCM, finishing products based on phosphorous were applied on the material containing the microcapsules, Pyrovatex ( $\mathrm{n}$-hydroxymethyl-3-dimethylphosphonopropionamide) and phosphoric acid. To complement the fire retardant treatment, Boric acid was first applied to the microcapsules as it could react with the MF wall of the PCM microcapsules.

The flame retardancy compared to the non-treated microcapsules was tested according to the standard test EN532 which included washing (5 times in washing machine). The washfastness was evaluated by weight measurement, before and after washing. The stiffness of the material was screened by different observers and only those materials which didn't alter too much their drape and tactile properties were accepted.

The thermal effect was tested in a prototype plate calorimeter.

\section{EXPERIMENTAL}

\section{Equipment}

The microcapsules' emulsion was applied in a Werner Mathis laboratory machine, the pressure being pneumatically controlled. Thermal fusion of double walled microcapsules and curing of melamine-formaldehyde resin was carried out in a Werner Mathis drying/curing chamber at $150^{\circ} \mathrm{C}$.

The calorimeter used was a prototype consisting of two plates through which a heat flux is produced. The top plate is heated to $45^{\circ} \mathrm{C}$ and the bottom plate kept at $25^{\circ} \mathrm{C}$. The heat sensor in the bottom plate measures the heat flux through the non-woven placed between the plates[4].

\section{Starting Materials}

Non-Woven (needled) supplied by Duflot Industries (France)

Fibre: Nomex (Aramid)

Mass: $110 \mathrm{~g} / \mathrm{m} 2$

Microcapsules of octadecane PCM with a Melamine-formaldehyde shell were supplied by Micropolis (Portugal).

The melamine-formaldehyde precondensate was supplied by BASF (Lyofix MLF and Kaurit TX). Pyrovatex was supplied by CIBA (Pyrovatex CP new)

\section{FIRE RETARDANT MICROCAPSULES}

mPCM coated with PMMA-PPBBA co-polymer

Microcapsules were coated with PMMA (polymethylmethacrylate) copolymerized with a fire retardant monomer, PPBBA (poly-pentabromobenzylacrylate) by radical polymerisation around the MF microcapsules of PCM [5].

\section{mPCM coated with PMMA-PMA co-polymer treated with zinc oxide}

A copolymer of PMMA with PAM (polymethacrylic acid) coated in the same way as above onto the MF mPCM, was treated with zinc oxide so as to attach zinc ions onto the carboxylic groups of the PAM polymer.

mPCM with Boric acid 
Boric acid was applied to the PCM microcapsules, and the MPCM were subsequentely washed and applied to the non-woven.

RESULTS FOR ARAMID NON-WOVEN CONTAINING MPCM

SEM photographs of Non-woven containing MPCM treated with Boric Acid

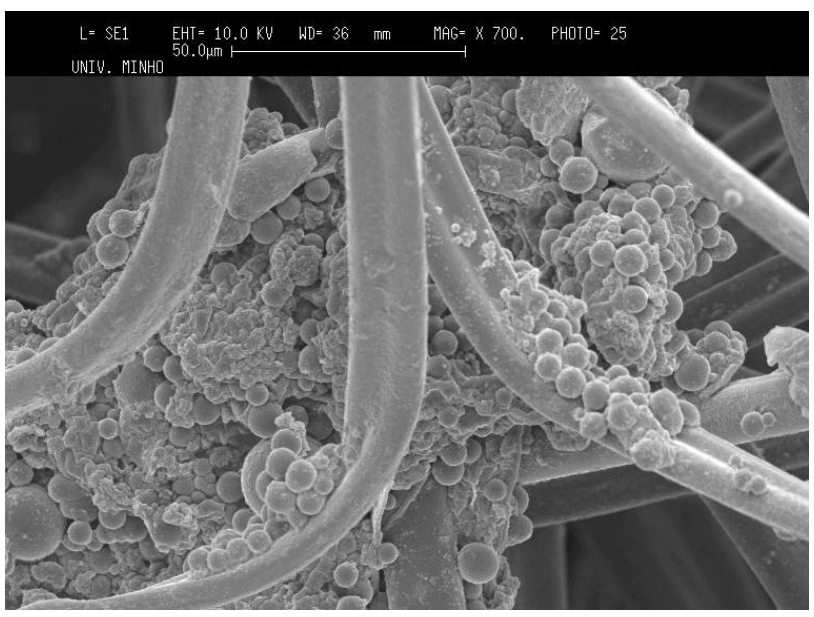

\section{Thermal Effect of non-woven}

A Thermograph for Aramid Non-woven was taken in the plate calorimeter, first for the sample without $\mathrm{mPCM}$ and then for comparison, on the same graph, for the non-woven containing double walled $\mathrm{mPCM}$, fire retardant (pyrovatex) and melamine-formaldehyde resin. The time the flux takes to reach half the maximum flux was measured. The graph is on figure 2 and the results for the flux are on table 1.

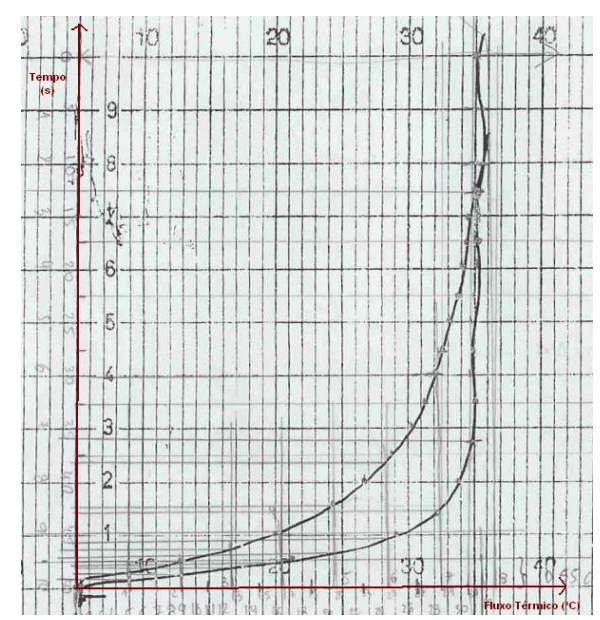

Fig.1. Thermograph for non-woven containing double walled mPCM

Table 1. Half-time of flux

\begin{tabular}{|l|l|}
\hline Sample & $1 / 2$ time \\
\hline Non-woven & 13.2 \\
\hline Non-woven with double & 26.2 \\
\hline
\end{tabular}




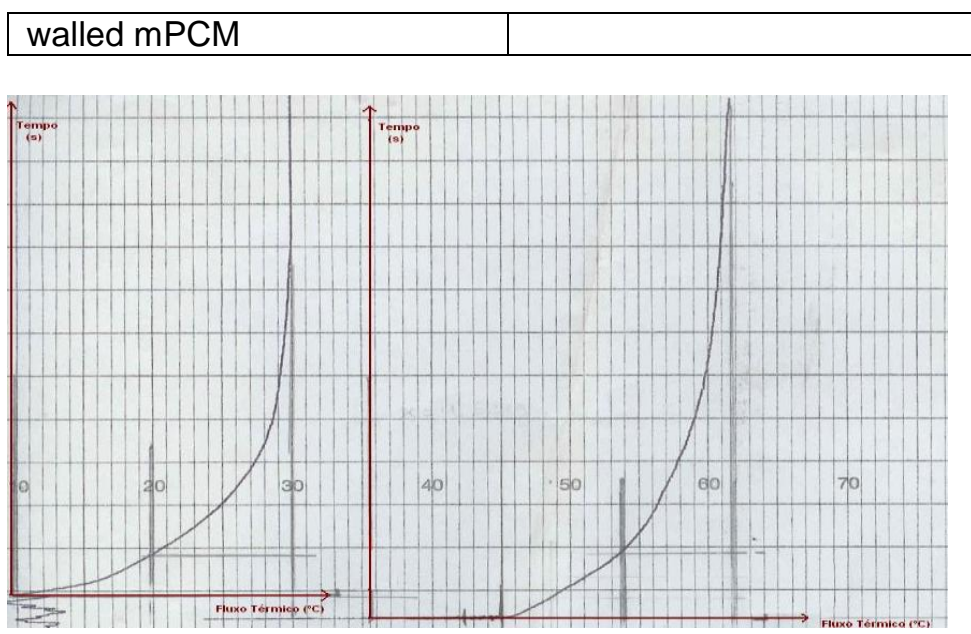

Fig. 2 Thermograph for non-woven containing single walled mPCM treated with Boric Acid

Table 2. Half-time of flux for non-woven containing singe walled mPCM treated Boric Acid

\begin{tabular}{|l|l|}
\hline Sample & $1 / 2$ time \\
\hline Non-woven & 10 \\
\hline $\begin{array}{l}\text { Non-woven with double } \\
\text { walled mPCM }\end{array}$ & 23 \\
\hline
\end{tabular}

\section{WASHFASTNESS RESULTS}

Double walled microcapsules

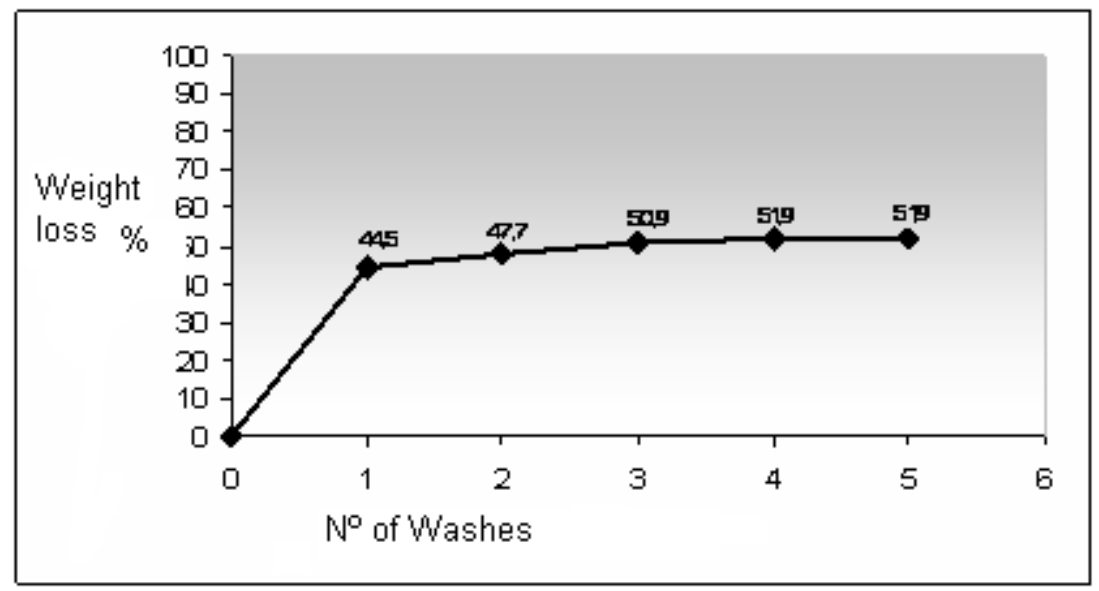

Fig.3. Weight loss of non-woven with double walled aPCM after 5 washes 
Single walled microcapsules with boric acid

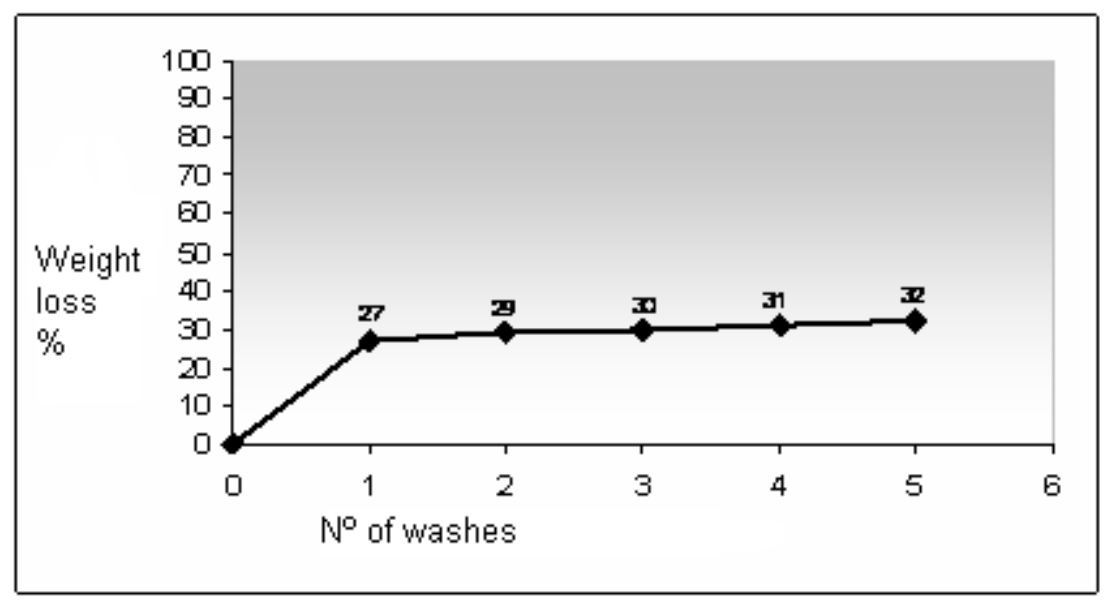

Fig 4. Weight loss of non-woven containing single walled MF MPCM treated with boric acid

\section{FLAME-RETARDANCY RESULTS}

The non-woven samples with different types of double walled mPCM were tested according to standard EN532. The results are on table 3.

\section{Table 3. Flame retardancy double walled PCM}

\begin{tabular}{|l|l|l|l|}
\hline Microcapsules & $\begin{array}{l}\text { Speed of flame } \\
\text { propagation }\end{array}$ & $\begin{array}{l}\text { Time of burning } \\
\text { (seconds) }\end{array}$ & Extent of burning \\
\hline Double walled mPCM & Fast & $>5$ & Total \\
\hline $\begin{array}{l}\text { Double walled mPCM } \\
\text { with PMBBA }\end{array}$ & Fast & $>5$ & Partial \\
\hline $\begin{array}{l}\text { Double walled mPCM } \\
\text { with Zinc }\end{array}$ & Medium & $3-5$ & Partial \\
\hline
\end{tabular}

The non-woven samples containing a flame retardant mixture of Pyrovatex (n-hydroxy-methyl-3dimethylpropionamide)+phosphoric acid and two types of MF MPCM were tested according to standard EN532. The results are on table 4.

Table 4. Flame retardancy of MF mPCM

\begin{tabular}{|l|l|l|l|}
\hline Microcapsules & $\begin{array}{l}\text { Speed of flame } \\
\text { propagation }\end{array}$ & $\begin{array}{l}\text { Time of burning } \\
\text { (seconds) }\end{array}$ & Extent of burning \\
\hline MF mPCM & Fast & $>5$ & Total \\
\hline $\begin{array}{l}\text { MF mPCM in non-woven } \\
\text { with Pyrovatex+phosphoric } \\
\text { acid }\end{array}$ & None & 0 & None \\
\hline mPCM with Boric Acid in & None & 0 & None \\
\hline
\end{tabular}


non-woven with Pyrovatex +

phosphoric acid

\section{TACTILE (HANDLE), DRAPE (rigidity) AND OTHER PROPERTIES OF MATERIALS}

Other properties important for the commercial viability of the material, such as tactile properties (handle), adhesion of microcapsules when shaking and applying friction to the material, other noticeable characteristics such as smell, were evaluated by independent observers. The results are on table 5 .

Table 5. Properties of Non-woven material containing microcapsules

\begin{tabular}{|l|l|l|l|}
\hline Microcapsules & Handle & Rigidity & Other \\
\hline $\begin{array}{l}\text { Non-woven with Double } \\
\text { walled mPCM treated with } \\
\text { zinc oxide }\end{array}$ & harsh & Medium & $\begin{array}{l}\text { Low adhesion of } \\
\text { mPCM to fibres }\end{array}$ \\
\hline $\begin{array}{l}\text { MF mPCM in non-woven } \\
\text { with Pyrovatex+phosphoric } \\
\text { acid }\end{array}$ & Medium & Hard & Strong odour (fish) \\
\hline $\begin{array}{l}\text { mPCM with Boric Acid in } \\
\text { non-woven with Pyrovatex }+ \\
\text { phosphoric acid }\end{array}$ & Soft & $\begin{array}{l}\text { Low (High } \\
\text { drape) }\end{array}$ & None \\
\hline
\end{tabular}

\section{DISCUSSION OF THE RESULTS}

Treated double walled microcapsules were not sufficiently flame-retardant to withstand the standard test EN532 as can be seen in table 3 and figure 3.Single walled MF mcirocapsules bound by MF resin and non-woven treated with flame retardancy products, resisted the flame test EN532, as seen in table 4, but altered considerably the properties of the original non-woven material with respect to handle and rigidity. They also released a strong unpleasant fish odour, due to the large quantity of Pyrovatex needed for withstanding the flame test.

When this product was replaced partially by Boric acid, these properties improved a lot. Boric acid gave the best results and did not wash-off from the microcapsules nor from the non-woven material. The reason must be that they react with both the microcapsules MF wall and with the MF resin, according to the equation:

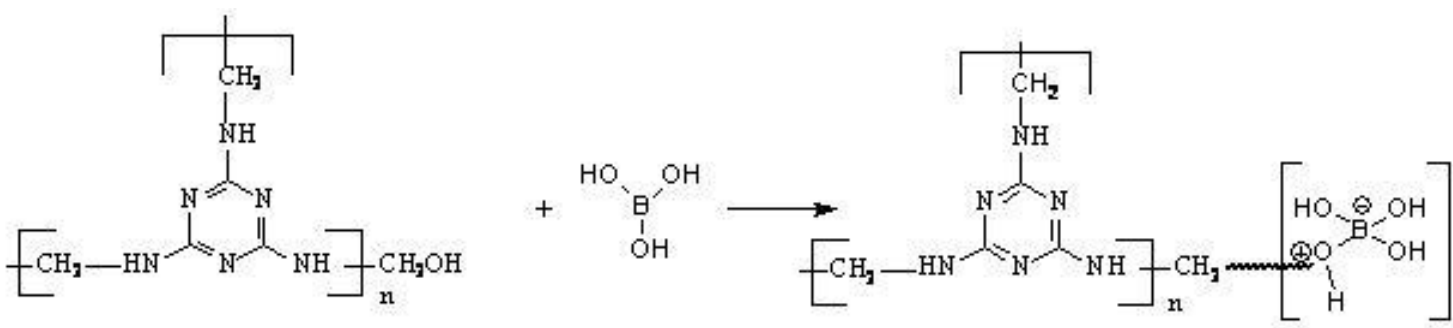

The washfastness of the single walled MF microcapsules with Boric acid, bound by MF resin and with Pyrovatex and phosphoric acid, also gave the best results for washfastness which suggests that there was a reaction between the MF wall of the MPCM, the MF resin and the nhydroxy-methyl-3-dimethylphosphonopropionamide (Pyrvatex).

\section{CONCLUSIONS}

For achieving the flame retardancy standard in a non-woven lining inside firefighters' protective clothes, of the different technologies tested, it was found that only a conjugation of both 
microcapsule protection and non-woven flame retardant finishing, was appropriate. It was also found that even the use of small quantities of thermoplastic binder around the microcapsules was sufficient for the material to fail the test. Boric acid previously applied on the mPCM was found to be a good complement to the standard flame retardancy finishing based on phosphorous and applied on the non-woven. In this way tactile and other properties of the original non-woven material were preserved.

\section{REFERENCES}

1. Kim, J. and Cho, G., Textile Res. J., 2002, 72(12), 1093-1098.

2. Choi, K, Cho, G., Kim, P. and Cho, C., Textile Res. J., 2004, 74, 292-296.

3. Su, J., Wang, L., Ren, L., Huang, ., J Appl Polym Sci, 2007, 103, 1295

4. Pushaw, J., US Patent 1997, US5677048.

5. Gomes, J., patent GB2417495, 18 April, 2007

\section{Corresponding Author:}

Jaime Rocha Gomes can be contacted at rgomes@det.uminho.pt 